\title{
A Simplified LuGre Model
}

\author{
Guo Jing-hao \\ School of Instrumentation Science and Optoelectronics \\ Engineering \\ Beihang University \\ Beijing, China \\ 1527882107@qq.com
}

\author{
Dong Quan-lin \\ School of Instrumentation Science and Optoelectronics \\ Engineering \\ Beihang University \\ Beijing, China \\ dongquanlin@buaa.edu.cn
}

\begin{abstract}
In the modern control system, especially the mechanical servo system, a suitable mechanical friction model is very important for the simulation verification of the control system or algorithm. At present, the traditional Dahl model has many defects in fitting the real friction and the simulation of the LuGre model which fits the real better is much more complicated. In this paper, a simplified LuGre model is established based on the characteristics of the Dahl model and the LuGre model. This model can be implemented with only a few simulink modules. Besides, by comparing with the simulation results of some other models, the fitting effect of the simplified model on the actual friction is closer to that of the LuGre model.
\end{abstract}

Keywords-friction model; Dahl model; LuGre model; Stribeck effect; Stiction; simulation of Stick-slip model

\section{INTRODUCTION}

Friction is ubiquitous in the modern industry. In some cases, friction is beneficial to our production activities, such as vehicle walking, conveyor belts, etc., and in some cases, friction is a serious obstacle to the further development of our industrial production system. In the modern control system, the friction will cause not only the dead zone and the limit cycle phenomenon in the position tracking system, but also crawling, oscillation and other phenomenon in the speed servo system ${ }^{[1]}$. The oscillation of the circuit in the mechanical system will lead to the wear of the mechanical structure of the system, reduce the system life, and even affect the system performance parameters ${ }^{[2]}$. Therefore, it is very important to study the friction problem in the mechanical system, especially the nonlinear problem.

In the study of friction, how to establish the appropriate friction model has been an important research direction in modern tribology and engineering control. The establishment of suitable friction model not only helps to analyze the mechanism and influencing factors of friction in the system, but also helps to predict the operating state of the system under friction and to compensate for the problem caused by friction.

The friction in the mechanical control system is a complex phenomenon, so the establishment of the friction model has also gone through a long process, and a lot of friction models are established for a variety of different conditions. Popularly used friction models are Stribeck model, Karnopp model, Dahl model and LuGre model. But the Stribeck model, a function of relative velocity, still remains in the static model stage, while the Karnopp model does not determine the zero-speed range

\footnotetext{
National Science and Technology Support.
}

method, can't accurately describe the low relative speed in the case of friction ${ }^{[1]}$. The Dahl model proposed in the $1960 \mathrm{~s}$ compares the friction between the interfaces as a spring deformation process and describes the frictional force as a dynamic process. At present, the Dahl model is still a widely used model in the study of friction phenomena. However, this model can't describe the Stribeck effect represented mainly by the negative slope of the friction curve at low relative velocity. The LuGre model proposed in 1995 is based on the Dahl model. Based on the Dahl model spring theory, LuGre model describes the friction between the interfaces as the contact and separation process of the elastic bristles. This model can accurately describe the crawling, the limit oscillations, the pre-slant deformation, and the static Stribeck effect in the rubbing process $^{[3]}$. Although the LuGre model can describe the actual friction phenomenon more closely, the simulation of the model is more complex.

In this paper, referring to the document [4,5] and the representation method of mane variables in the Dahl model, simplified the LuGre model to the linear model, so that the model can be implemented with several simulink modules. Then by comparing with the simulation results of some other models, the availability of the simplified model will be demonstrated.

\section{MODEL ESTABLISHMENT}

The general differential form of the Dahl model is:

$$
\left.\begin{array}{c}
\frac{d z}{d t}=\sigma_{0}\left(v-\frac{|v|}{F_{c}} F\right) \\
F=\sigma_{0} z
\end{array}\right\}
$$

where $F$ is the friction force $N), F_{c}$ is the Coulomb friction $(N), \sigma_{0}$ represents the stiffness factor $(N / m)$.

The traditional Dahl model can't describe the static friction and Stribeck effect. As shown in Fig. 1, it can be seen that when the initial friction $F(0)$ is less than Coulomb friction $F_{c},|F|$, the friction of the slider, will never exceed $F_{c}{ }^{[1]}$, which leads to the use of Dahl model in the lag-slip motion simulation, can't reflect the lag-slip process. 


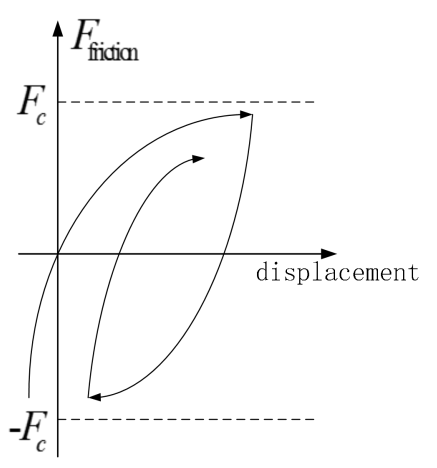

Fig. 1. Relationship between friction and displacement in Dahl effect

At present, the improvement of Dahl model in the literature is that the applicable interval of static friction is extended to a zero speed range, which makes Dahl model to be able to describe the characteristics of static friction.

$$
\left.\begin{array}{c}
\frac{d z}{d t}=v\left(1-\frac{h(|v|) \operatorname{sgn}(v)}{F_{s}} F\right) \\
F=\sigma_{0} z+\sigma_{1} \frac{d z}{d t}+\sigma_{2} v
\end{array}\right\}
$$

where $h(|v|)=\left\{\begin{array}{ll}1 & \text { if }|v| \leq \Delta v \\ \frac{F_{s}}{F_{c}} & \text { if }|v|>\Delta v\end{array}, \Delta v\right.$ is the zero speed interval limit.

Based on the Dahl model, the LuGre model adopts the idea of mane model, and introduces the average deformation of mane $z$. The function of the model can be expressed as:

$$
\left.\begin{array}{c}
F=\sigma_{0} z+\sigma_{1} \frac{d z}{d t}+\sigma_{2} v \\
\frac{d z}{d t}=v-\frac{|v|}{g(v)} z \\
g(v)=\frac{1}{\sigma_{0}}\left[F_{c}+\left(F_{s}-F_{c}\right) e^{-\left(\frac{v}{v_{s}}\right)^{2}}\right]
\end{array}\right\}
$$

where $\sigma_{1}$ is the damping coefficient $(N s / m)$ and $\sigma_{2}$ is the viscous friction coefficient $(N s / m)$.

The simplified LuGre model uses the representation of micro-displaceme $z$ in the general improved Dahl model, which makes the negative slope exponential attenuation of friction in the LuGre model linearized. Then it can be expressed by the following formula:

$$
\left.\begin{array}{c}
\frac{d z}{d t}=v\left(1-\frac{s(|v|) \operatorname{sgn}(v)}{F_{s}} F\right) \\
F=\sigma_{0} z+\sigma_{1} \frac{d z}{d t}+\sigma_{2} v
\end{array}\right\}
$$

where $s(|v|)=\left\{\begin{array}{c}\frac{|v|}{\Delta v}\left(\frac{F_{s}}{F_{c}}-1\right)+1 \quad \text { if }|v| \leq \Delta v \\ -\sigma_{2}|v|+\frac{F_{s}}{F_{c}}+\sigma_{2} \Delta v \text { if }|v|>\Delta v\end{array}, \Delta v\right.$ is the zero speed interval limit.

The friction characteristics of the model are shown in Fig. 2

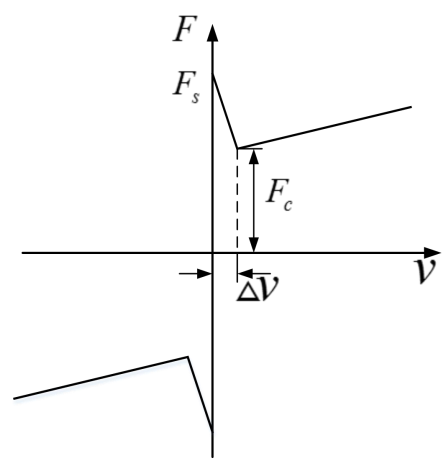

Fig. 2. Friction behaviour

\section{THE SIMULATION OF MODELS}

A classic hysteresis-slip algorithm is used to simulate the models mentioned above. Fig. 3 shows the model diagram of the example, in which the slider mass is constant and one end of the spring, whose elasticity is $k$, is pulled at a constant speed $v_{p}$. Since the slider is subjected to friction and the static friction is greater than the dynamic friction, the motion of the slider will show the phenomenon of stagnation and sliding state alternately ${ }^{[6]}$.

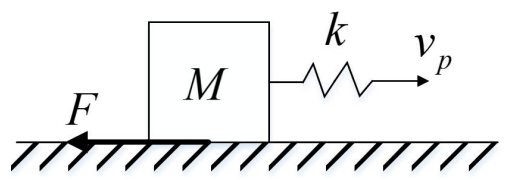

Fig. 3. Stick-slip experiment

The simulation results of the traditional Dahl model are shown in Fig. 4. The traditional Dahl model can't describe the stagnation-slip phenomenon well while simulating the hysteresis-slip motion. From Fig. 4-b, it can be seen that the Dahl model can't describe the static frictional force and the Stribeck effect ${ }^{[1]}$, and there is a slight oscillation in the relationship between friction and velocity. 


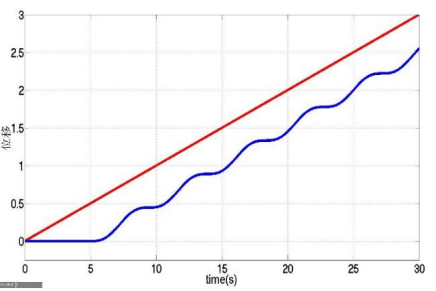

a. Relationship between

displacement and time

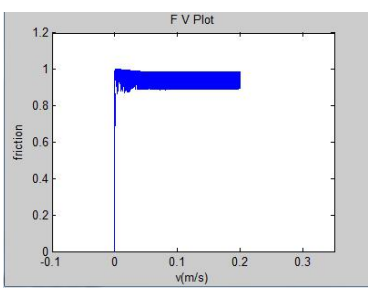

b. Relationship between

friction and speed
Fig. 4. Simulation result of Dahl model

The simulation results of the general improved Dahl model are shown in Fig. 5. And it shows that the model can describe the hysteresis-slip process. Moreover, in the friction and velocity function images of Fig. $5-b$, it is seen that there is no slight concussion any more in the the general improved Dahl model simulation, but the model can't describe the Stribeck effect at low velocity, either.

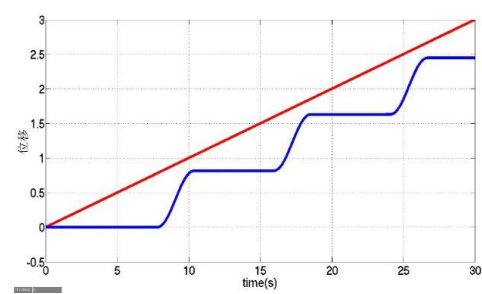

a. Relationship between displacement and time

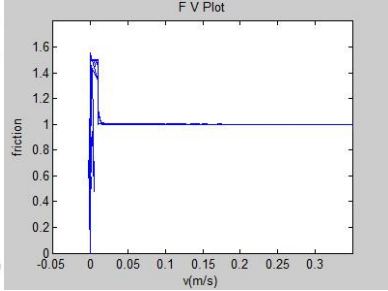

b. Relationship between friction and speed
Fig. 5. Simulation result of general improved Dahl model

Fig. 6 shows that the LuGre model can describe the stagnation-slip process and reflect the Stribeck effect at low speed.

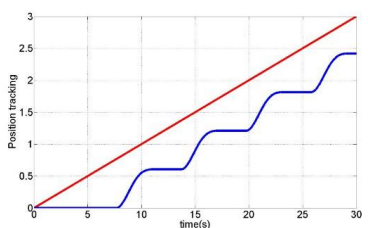

a. Relationship between

displacement and time

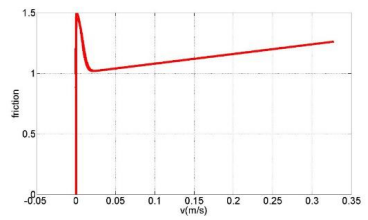

b Relationship between friction and speed
Fig. 6. Simulation result of LuGre mode

Fig. 7 shows that the system can be implemented with only a few simulink modules. The simulation results of the simulation block diagram are shown in Fig. 8.

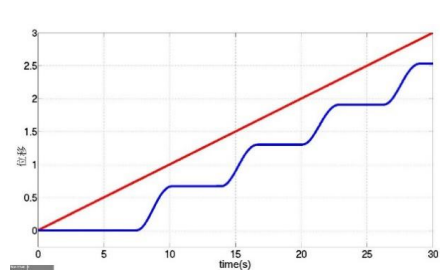

a Relationship between

displacement and time

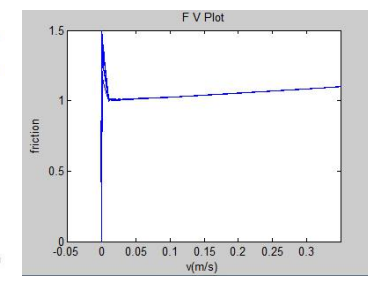

b Relationship between

friction and speed
Fig. 7. Simulation result of the simplified LuGre mode

As shown in Fig. 8, it can be seen that the simplified LuGre model can approximate the Stribeck effect. Besides, the model can reflect the static friction force and the slack-slip motion of the slider. Moreover, in the stagnation-slip process of the simulation slider, for a LuGre model, a hysteresis-slip period is approximately $6.12 \mathrm{~s}$, and in the simulation of the general improved Dahl model under the same conditions, due to the decrease from static friction to sliding friction only happens at the speed threshold, it is necessary to go through a period of about $8.15 \mathrm{~s}$. However, using the simplified model, a hysteresisslip period is about $6.52 \mathrm{~s}$ in the motion of the slider. It can be seen that the results of the simplified LuGre model simulation is closer to that of the LuGre model.

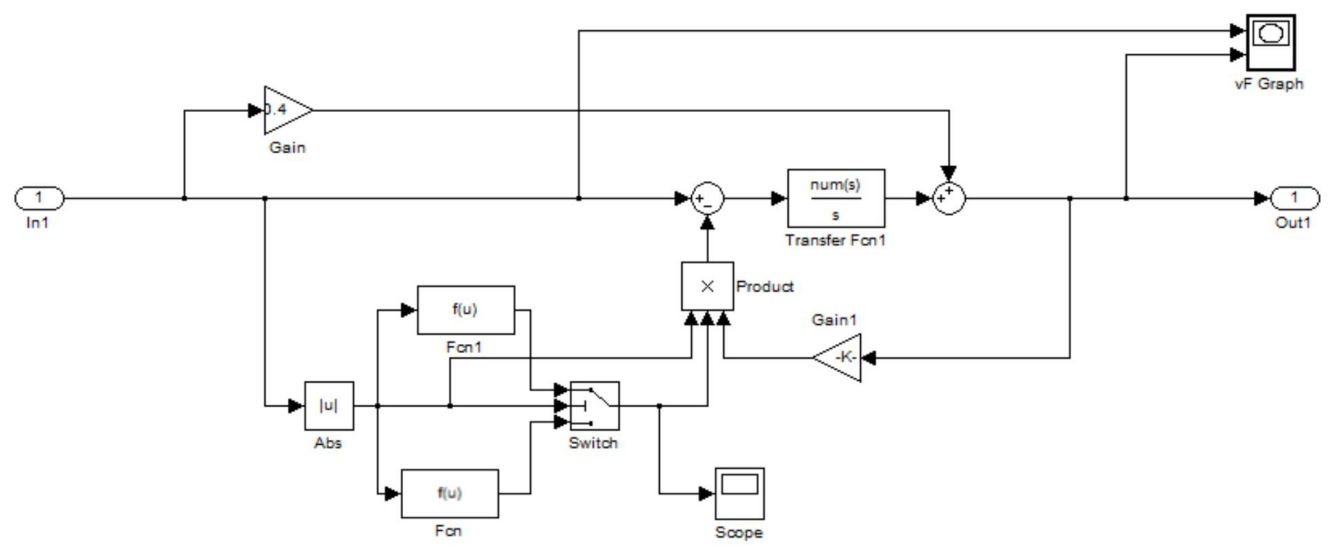

Fig.8. Simulation diagram of the simplified LuGre model 


\section{SUMMARY}

In this paper, the simplified LuGre friction model is established, and the simulation results are compared with the Dahl model, the general improved Dahl model and the LuGre model.The following two analysis results are obtained:

(1) The simplified LuGre model can describe the characteristics of static friction, Coulomb friction and viscous frictional force in the simulation of stagnation-slip motion and the model can approximate the Stribeck effect. In the case of the same control parameters, the simulation results of the model is closer to that of the LuGre model, compared with the Dahl model and the general improvement scheme.

(2) The simulation of the simplified LuGre model is simpler than that of the LuGre model, and it can be realized by just a few simulink modules. In the case of the same control parameters, the simulation results of the model is closer to the LuGre model's , compared with the Dahl model and the general improvement scheme.
From the above two points, it can be seen that the simplified LuGre model may provide some reference significance and application value for the research and compensation of friction in mechanical servo system.

\section{REFERENCES}

[1] Liu Guo-ping. Friction model and simulation in mechanical system[D]. Xi'an: Xi'an University of Technology, 2007(In Chinese)

[2] Li Shi-zhe, Wang Yin-song, Tian Jing-yu. Simulation of pneumatic valves stiction and analysis of control system oscillation[J]. Computer Simulation, 2016, 33(1): 239-244(In Chinese)

[3] Zhang $\mathrm{Hu}$, Hu Hai-na, Wang Wu. Simulation of fuzzy sliding mode control for servo turntable friction system[J]. Machinery Design \& Manufacture, 2012, 3: 174-176(In Chinese)

[4] Wang Yi, He Zhen, Wang Guang-xiong. A practical friction model[J]. Electric Machines and Control, 2011, 15(8): 59-63(In Chinese)

[5] Liu Na, Mei Zhi-qian, Li Xiang-guo, Wang Rui-juan. Research on adaptive friction compensation based on modified Dahl model[J]. Journal of Mechanical and Electrical Engineering, 2012, 29(12): 13911394,1409(In Chinese)

[6] ASTROM K J, CANUDAS-DE-WIT C. Revisiting the LuGre friction model[J]. IEEE Control Systems Magazine, 2008, 28(6): 101-114. 